\title{
Medical Image of the Week: Leptomeningeal Carcinomatosis
}
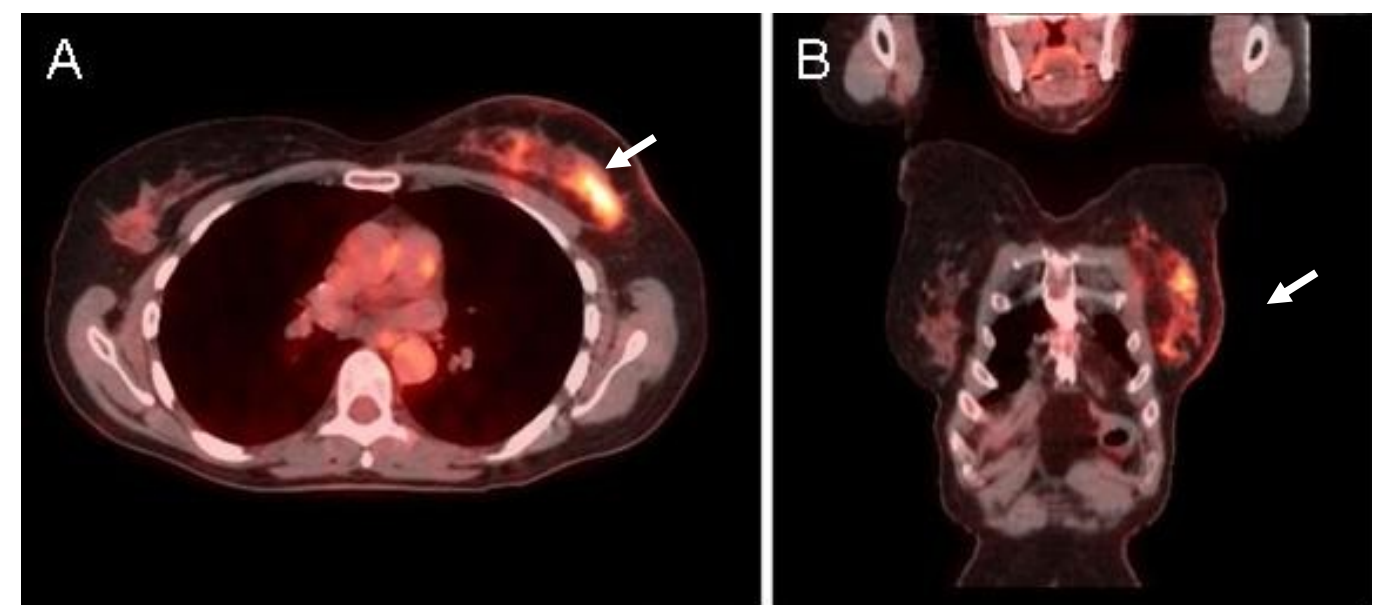

Figure 1. PET/CT scan showing metabolic activity in the mid $3^{\text {rd }}$ of the left breast without significant adenopathy (arrows).

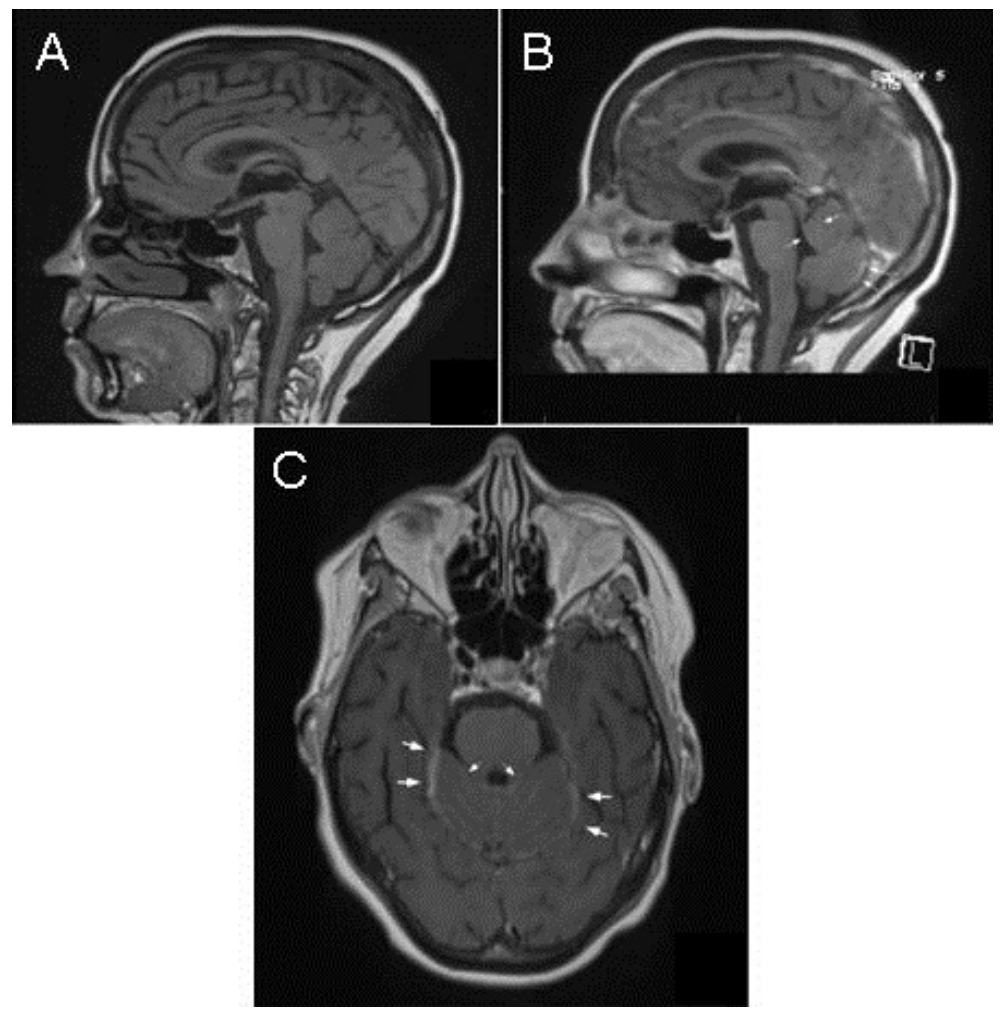

Figure 2. Panel A: Brain MRI T1 pre contrast image shows normal signal intensity in cerebrum, brain stem and cerebellum with no evidence of acute infarction. Panels B and C: Post contrast images show diffuse leptomeningeal enhancement involving bilateral cerebellar folia and around the surface of the brainstem (arrows). 
A 65 year old woman with a history of breast cancer presented to the emergency department (ED) with dizziness and disequilibrium, which started a week prior to admission. A year ago, she was diagnosed with locally advanced lobular carcinoma confined to the left breast (Figure 1). She underwent mastectomy followed by chemoradiation including taxol, sunitinib, cyclophosphamide and doxorubicin with remarkable response, and achieved complete remission. In the ED, her neurologic status deteriorated rapidly, she developed tonic-clonic seizures and became unresponsive to verbal and painful stimuli. CT of the head showed no evidence of acute intracranial abnormality or metastatic lesion, however, a brain MRI brain showed contrast enhancement and increased fluid attenuated inversion recovery (FLAIR) signal of the leptomeninges in cranial nerves III, V, VII and VIII as well as cerebellar surface, suggesting meningeal carcinomatosis (Figure $2 \mathrm{~B}$ and $2 \mathrm{C}$ ). A lumbar puncture demonstrated malignant cells in the cerebospinal fluid confirming the diagnosis of leptomeningeal carcinomatosis. Palliative radiation therapy with thiotepa was planned, however, her family opted for comfort care only and the patient passed away $6^{\text {th }}$ day of hospital stay.

Leptomeningeal carcinomatosis (LC) is a devastating complication of systemic cancer that can occur in patients with solid or hematologic malignancies. LC has been described in $5 \%$ to $10 \%$ of patients with solid tumors, more frequently breast adenocarcinoma, lung adenocarcinoma and melanoma $(1,2)$. LC may be the first manifestation of cancer in $5 \%$ to $11 \%$ of patients and maybe the sole site of relapse in patients successfully treated for cancer (2). Treatment of LC is currently palliative for most patients, with an expected median survival of less than 6 months $(1,2)$

Roberto Bernardo MD, Seongseok Yun MD PhD, Ateefa Chaudhury MD, Keri Maher DO, and Tauseef Siddiqi MD

Department of Medicine, University of Arizona, Tucson, AZ

\section{References}

1. Bruna J, González L, Miró J, Velasco R, Gil M, Tortosa A. Leptomeningeal carcinomatosis: prognostic implications of clinical and cerebrospinal fluid features. Cancer. 2009;115(2):381-9. [CrossRef] [PubMed]

2. Kesari S, Batchelor TT. Leptomeningeal metastases. Neurol Clin. 2003:21(1): 25-66. [CrossRef] [PubMed] 